\title{
Änderungen im Chemismus des Interstitialwassers am Strand von Cuxhaven während einer Tide
}

\author{
HeINRICH KüHL und Hans ManN ${ }^{1}$ \\ Bundesforschungsanstalt für Fischerei, Institut für Küsten- und \\ Binnenfischerei, Hamburg
}

\begin{abstract}
Changes in the chemistry of the interstitial water at the beach of Cuxhaven during a tide. At the "Muschelecke" near our Cuxhaven Laboratory (North Germany), salinity, $\mathrm{O}_{2}$-content, nitrate, phosphate, alkalinity, as well as lime and $\mathrm{Mg}$ contents were determined during one tide in the interstitial water and the free water above. Interstitial salinity was always lower than in the free water; $\mathrm{O}_{2}$-content decreased with increasing depth below the tide mark. Nitrate and phosphate contents as well as alkalinity were higher, lime and magnesium contents lower than in the free water.
\end{abstract}

\section{EINLEITUNG}

Aus Untersuchungen an den Stränden von Seen und Flüssen ist bekannt, daß das interstitielle Wasser des Ufers sich in seinen Eigenschaften von dem des freien Gewässers unterscheidet. WISZNIEWSKI (1934) und STANGENBERG (1934) fanden, daß dies nicht nur für das Gebiet des untergetauchten Sandes gilt, sondern ebenso für das Sandlückensystem oberhalb der Wasserlinie. Die Tierwelt dieses Gebietes, des Mesopsammals, ist durch zahlreiche Untersuchungen im Süß- und Seewasser schon gut bekannt, während unsere Kenntnisse über den Chemismus des Lückenwassers noch verhältnismäßig gering sind. Von St'ANGENBERg (1934) und Wiszniewskr (1934) wurde herausgestellt, daß das Lückenwasser ein besonders eutrophes Milieu darstellt. Schwierigkeiten für die Untersuchungen ergeben sich daraus, daß die Horizonte der verschiedenen Wasserschichten oft sehr klein sind, worauf GERLACH (1953) schon hingewiesen hat.

In früheren Untersuchungen am Sandstrand von Cuxhaven und Helgoland konnten wir nachweisen, daß sich das Porenwasser in einem reinen Sandgebiet ohne Anwurf nur wenig vom freien Wasser unterscheidet; ist jedoch Angespül vorhanden, so können beträchtliche Unterschiede in den chemischen Faktoren auftreten. Diese Ergebnisse wurden in Schnitten gewonnen, die von der Wasserkante in gewissen Abständen strandaufwärts entnommen wurden. Das Wasser wurde aus gegrabenen Löchern unterschiedlicher Tiefe entnommen. Auf diese Weise erhielten wir einen Einblick in die örtlichen Unterschiede der chemischen Verhältnisse (KüHL \& MANN 1966).

1 Unserem gemeinsamen Freunde Dr. G. KuUst zum 60. Geburtstag gewidmet. 
Durch die Tidenbewegung ändert sich bekanntlich die Höhe des Wasserspiegels ganz beträchtlich. In Flußmündungsgebieten können gleichzeitig in Verbindung hiermit große Unterschiede im Salzgehalt und anderen chemischen Faktoren auftreten (KüHI \& MANN 1953). Es war daher zu prüfen, ob und in welchem Maße sich die chemischen Verhältnisse im Lückensystem im Zusammenhang mit den Gezeiten ebenfalls ändern. Besonders geeignet erschien uns hierfür das Strandgebiet in der Elbmündung bei Cuxhaven, da hier die abiotischen Faktoren normalerweise schon großen Schwankungen unterliegen (KüHL 1952, 1962).

\section{METHODIK}

In unmittelbarer Nähe des Cuxhavener Laboratoriums befand sich ein Sandstrand, auf dem in höheren Lagen sehr viel Muschelschill abgelagert war. Infolgedessen bestand der Strand aus einem Gemisch von Sand und Mytilus- und Cardium-Schill.

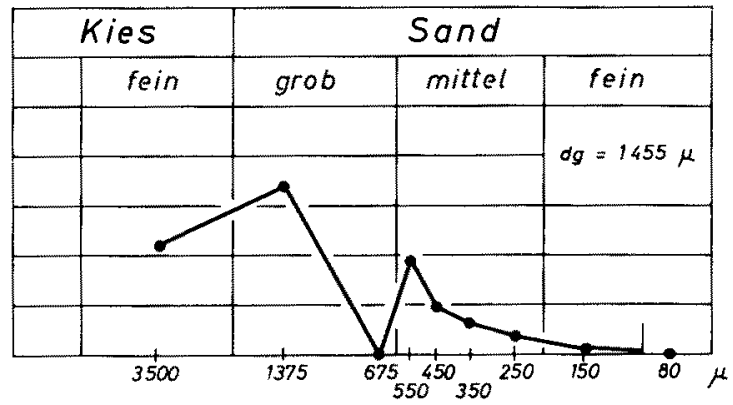

Abb. 1: Konverteilungskurve des Muschelschillsandes am Untersuchungsplat\%: „Muschelecke“ am Laboratorium Cuxhaven

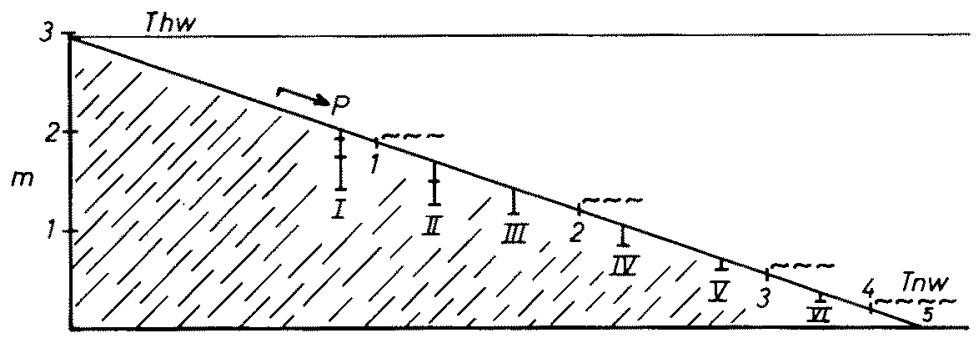

Abb. 2: Probeentnahmestellen an der "Muschelecke“. I bis VI Wasserproben aus gegrabenen Löchern in verschiedenen Tiefen (schwarze Säulen in Abb. 4-7); 1 bis 5 Probeentnahme aus dem "freien“ Wasser bei verschiedenem Wasserstand (weiRe Süulen in Abb. 4-7); Thw: Tidenhochwasser, Tnw: Tidenniedrigwasser, P: ablaufendes Porenwasser, Tidenhub (Differen\% zwischen Thw und Tow) $=2,93 \mathrm{~m}$

Die Durchschnittsgröße der Körner dieses Sandes betrug $1455 \mu$; die Kornverteilungskurve gibt Abbildung 1 wieder. Das Wasserhaltevermögen betrug 30 bis $35 \%$. Der Strand stieg gleichmäßig in einem Winkel von etwa $20^{\circ} \mathrm{C}$ an. 
An diesem Strand wurde ein Meter oberhalb der Wasserlinie ein Loch gegraben und das Sickerwasser aufgefangen. Dann wurden mit ablaufendem Wasser in Abständen von jeweils zwei Meter fünf weitere Löcher gegraben. Auf diese Weise gewannen wir sechs Untersuchungsstellen. Mit Absinken des Wasserspiegels bei Ebbe fielen die höher gelegenen Löcher trocken, dann wurden sie soweit vertieft, bis sich neues Sickerwasser ansammelte, das entnommen wurde. In der Zwischenzeit wurden zum Vergleich Wasserproben aus dem freien Wasser geschöpft (Abb. 2).

Die chemische Untersuchung erstreckte sich auf folgende Faktoren: Wassertemperatur, Salzgehalt, pH-Wert, Sauerstoffgehalt, Sauerstoffzehrung, Alkalität, Gehalt an Kalk und Magnesium, Ammonium, Nitrit, Nitrat und Phosphat. Die Bestimmung erfolgte nach den Deutschen Einheitsverfahren zur Wasseruntersuchung (1960). Die Bestimmung der Korngröße wurde dankenswerterweise in der Hydrographischen Abteilung des Instituts für Meeresforschung, Bremerhaven, bei Dr. LüNEBurg durchgeführt.

\section{ERGEBNISSE}

In Flußmündungsgebieten spielt der Salzgehalt des Wassers eine besondere Rolle. Aus diesem Grunde soll der Salzgehalt an erster Stelle abgehandelt werden. Alle Ergebnisse unserer Untersuchungen am Psammolitoral des Elbmündungsbereichs deuten

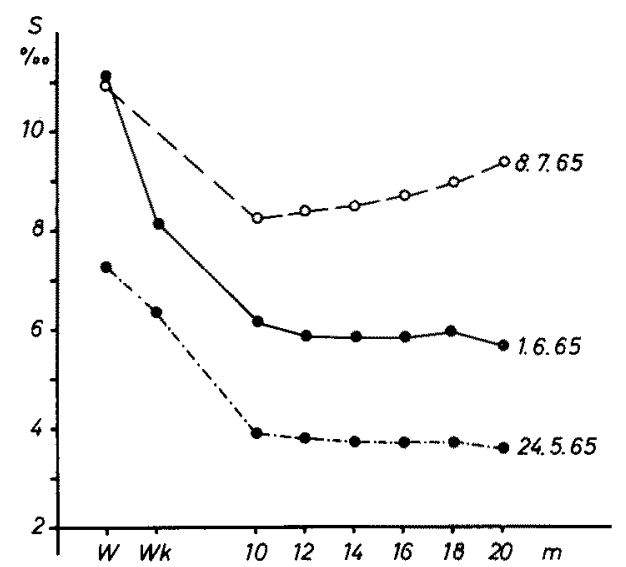

Abb. 3: Anderungen des Salzgehaltes des freien Wassers (W), der Wasserkante (Wk) und des Porenwassers in verschiedenen Entfernungen vom Spülsaum an einigen Untersuchungstagen mit unterschiedlichem Salzspiegel des Elbwassers

darauf hin, daß der Salzgehalt des freien Wassers höher ist als der des Porenwassers. In Abbildung 3 sind einige Beispiele früherer Untersuchungen bei unterschiedlichem Salzgehalt des Elbwassers dargestellt. Interessanterweise ist auch der Salzgehalt des Wassers in unmittelbarer Nähe des Spülsaumes niedriger als im freien Wasser. Noch geringer ist der Salzgehalt des Porenwassers. Dieses zeigt, wenn es in den verschiedenen Höhenlagen zu gleicher Zeit gewonnen ist, in sich nur geringe Schwankungen. Da bei 
Ebbe das salzärmere Porenwasser aus den Lückenräumen austritt und den Hang herunterläuft (Abb. 2, 8), tritt eine Verdünnung ein, die sich in dem niedrigeren Salzgehalt des Wassers an der Wasserkante bemerkbar macht. Wird das Porenwasser an einer Stelle $\mathrm{zu}$ verschiedenen Zeiten entnommen, so findet man hier doch gewisse Schwankungen, die durch das auflaufende oder ablaufende Wasser bedingt sind. Eine Untersuchung dieser Art ist in Abbildung 4 dargestellt, die zeigt, daß in den tiefer gelegenen Löchern V und VI der Salzgehalt höher ist als in den darüberliegenden Löchern, denn es tritt hier schon wieder eine Vermischung mit dem "Salz"-Wasser des freien Wassers ein. Auch sei darauf hingewiesen, daß diese tiefer gelegenen Löcher länger von Seewasser bedeckt sind. Die Erniedrigung des Salzgehaltes im Porenwasser

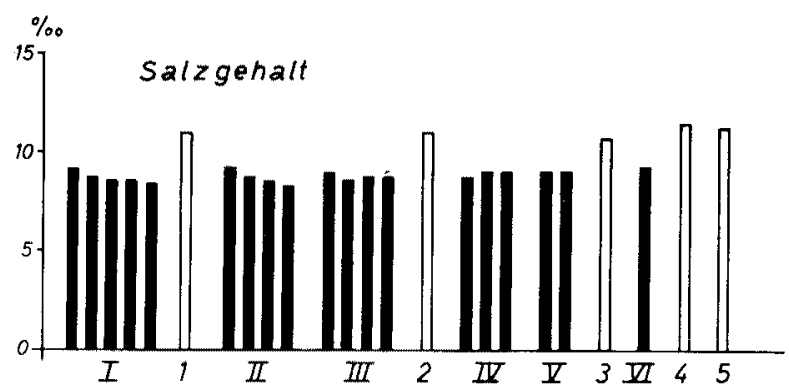

Abb. 4: Zeitliche Anderungen des Salzgehaltes während einer Untersuchung am 8. Juli 1965 in den gegrabenen Löchern I bis VI (vgl. Abb. 2) und des freien Wassers bei Ebbe (Nr. 1-5)

dürfte durch Regenwasser hervorgerufen scin. Wieweit Salzionen während der Passage durch den Sand zurïckgehalten werden, haben wir noch nicht untersucht (STOWELL 1927, RiEmanN 1966).

Ein weiterer lebenswichtiger Faktor ist der Sauerstoft. Anderungen im Sauerstoffgehalt von Gewässern können sehr schnell auftreten, wie Untersuchungen in Aquarien und Wattpfützen gezeigt haben (KürL 1952, 1962, KüHL \& MANN 1953, 1966). Bei unseren früheren Untersuchungen am Sandstrand von Helgoland konnten wir ebenfalls feststellen, daß rasche Veränderungen im Sauerstoffgehalt des Porenwassers auftreten können. Allgemein kann man sagen, daß der Sauerstoffgehalt in den höher gelegenen Bereichen günstiger ist. Er nimmt zum Wasser hin ab. Im Bereich des normalen Angespüls (Fucus, Muschelschalen, organische Drift) ist er am niedrigsten. In den oberen Bereichen traten auch die größten Schwankungen auf (Abb. 5). Sie können über $30 \%$ der Sättigung betragen. In den Lödhern (z. B. Loch III), wo der absolute Sauerstoffgehalt schon niedriger ist, hat die Schwankungsbreite naturgemäß auch einen geringeren Umfang. Im Bereich des Angespüls (Loch IV und V) ist der Gehalt, wie bereits angeführt, besonders niedrig und die Schwankung minimal. Der Sauerstoffgehalt am Spülsaum ist bei hohem Wasserstand bei oder kurz nach Tidenhochwasser höher als das Porenwasser, er geht mit zunehmender Ebbe etwas zurück, weil von oben her sauerstoffärmeres Wasser aus dem Lückensystem nachläuft und sich untermischt.

Recht interessant sind auch die Werte der Sauerstoffzehrung. Überblickt man das Gesamtbild der Zchrung, so stellt es cine gute Ergänzung zum Sauerstoffgehalt dar (Abb. 5 unten). Entsprechend der Abnahme des Sauerstoffgehaltes von den höher 
gelegenen Probeentnahmestellen zum Wasser hin nimmt die Zehrung in der gleichen Richtung $z u$. Sie steigt von $10 \%$ auf uber $50 \%$ an. Ahnliche Verhältnisse finden wir auch bei jedem einzelnen Punkt. Hier wird die Zehrung im Verlauf der Untersuchung mit abnehmendem Sauerstoffgehalt größer. Ausnahmen machen die zeitlich zuletzt stattfindenden Probeentnahmen, wenn das auflaufende Wasser mit der Flut wieder das

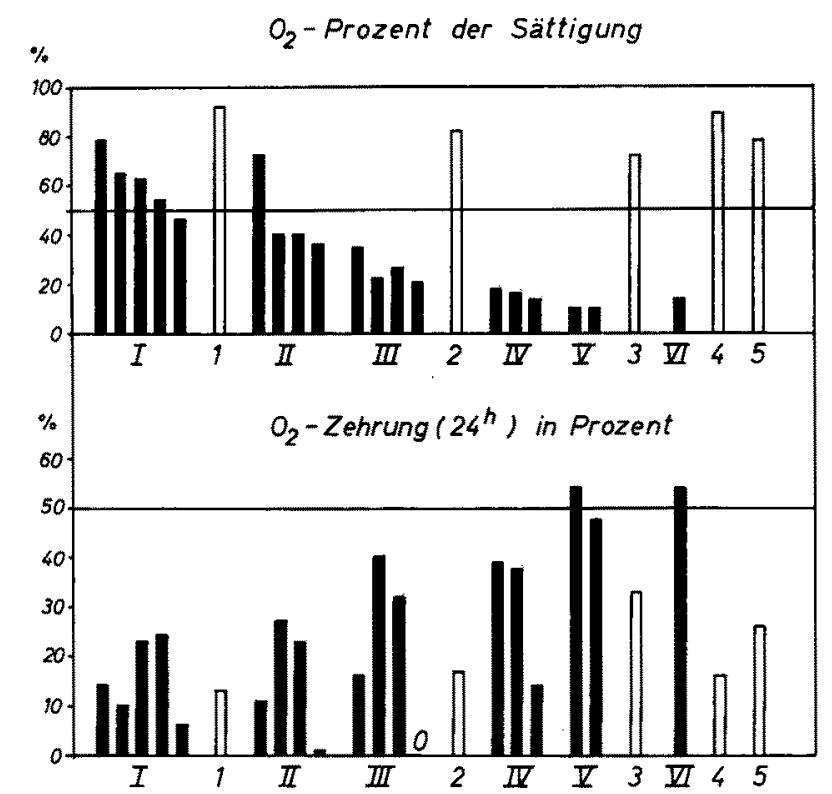

Abb. 5: Sauerstoffgehalt in Prozent der Sättigung (oben) und Sauerstoffzehrung nach 24 Stunden in Prozent (unten) an den Untersuchungsstellen (vgl. Abb. 4)

Niveau der Löcher erreicht. Mit diesem wird ein sauerstoffärmeres, aber weniger mit sauerstoffzehrenden Stoffen belastetes Wasser herangeführt. Möglicherweise findet auch eine Mischung mit Restwasser statt, das in dem Porensystem bei den vorhergehenden Abläufen zurückgeblieben ist.

Die Werte für den Ammoniumgehalt waren bei allen Untersuchungen so gering, daß sich eine Diskussion erübrigt. Nur im Bereich des Fucus und Angespïls wurden geringe Gehalte gefunden. Ahnliches gilt auch für das Nitrit, das wir mit Werten von $0,4 \mathrm{mg} / \mathrm{l} \mathrm{im}$ Bereich des Angespüls fanden. Ebenso wurden Nitrite mit Werten von 0,05 bis $0,3 \mathrm{mg} / 1$ an der Wasserkante unter dem Bereich des Angespüls nachgewiesen. Wie oben schon erwähnt, stammen diese Nitrite aus dem ablaufenden Porenwasser. Die Zahlenwerte für den Nitratgehalt liegen sehr dicht beieinander; man kann aber daraus entnehmen, daß der Nitratgehalt am gleichen Punkt mit zunehmender Tiefe etwas ansteigt. Das freie Wasser hat stets einen etwas geringeren Gehalt. Das Nitrat als Endprodukt der Nitrifikation hat sich im Boden angereichert, da vermutlich auch der Verbrauch dieses Nährstoffes im Bodenwasser nicht bedeutend ist. Es wird bei ablaufendem Wasser zum Teil ausgewaschen und erscheint dann im strandnahen freien Wasser (Abb.6). 
Größere Unterschiede finden wir bei den Phosphatwerten; sie bewegen sich zwischen 0 und $58 \gamma / 1$ an den verschiedenen Entnahmestellen. Das ablaufende Wasser an der Wasserkante ist bei Mittelwasser noch relativ phosphatreich. Der Gehalt an Phosphaten im ablaufenden Wasser geht im Laufe der Ebbe schnell auf niedrige Werte oder sogar Null zurück. Der Gehalt im Lückenwasser ist höher als im freien Wasser; die

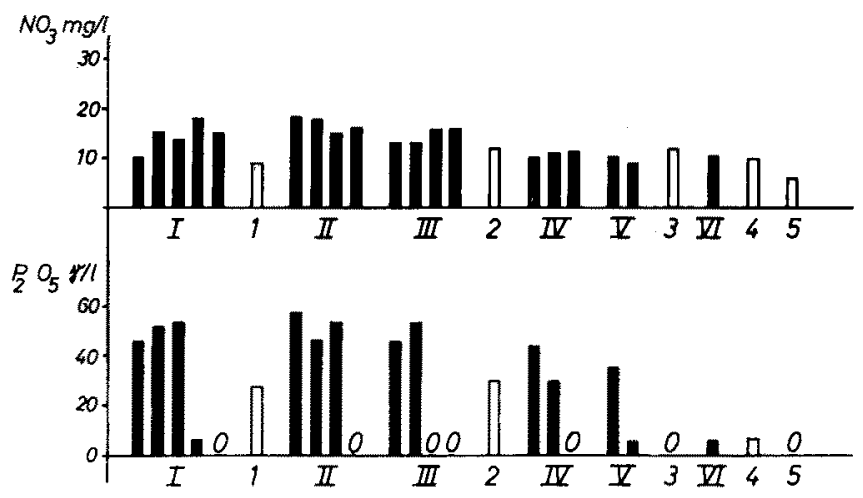

Abb. 6: Nitratgehalt (oben) und Phosphatgehalt (unten) an den Untersuchungsstellen (vgl. Abb. 4)

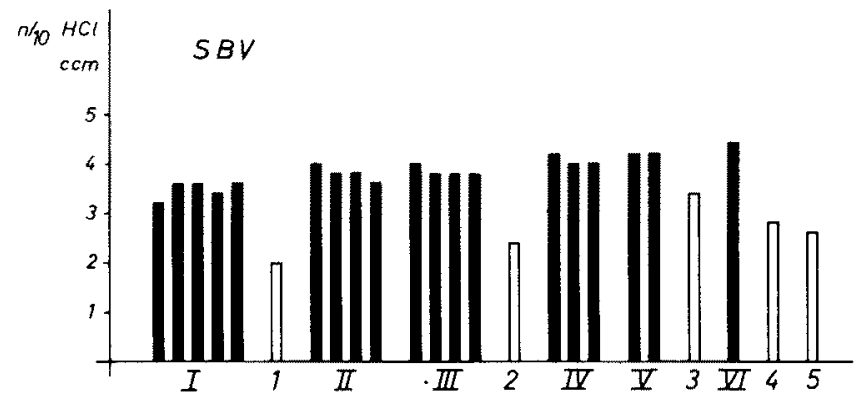

Abb. 7: Säurebindungsvermögen an den Untersuchungsstellen (vgl. Abb. 4)

Werte lagen bei $50 \gamma / 1$. Auf gleichem Horizont fanden wir in den jeweils $2 \mathrm{~m}$ entfernten Grablöchern den gleichen höchsten Wert von $55 \% / 1$. Bei Flut dringt phosphatarmes Wasser in das Lückensystem ein, und die Werte gehen dann zurück, teilweise bis auf Null.

Auch im Säurebindungsvermögen kommt noch einmal die allgemeine Tendenz zum Ausdruck, daß die höheren Werte im tieferen Bereich des Strandes liegen (Abb. 7). Selbstverständlich hat das Lückenwasser $(3,2$ bis $4,4 \mathrm{ccm} \mathrm{n/10} \mathrm{HCl}$ ) das größere Säurebindungsvermögen als das freie Wasser $(2,0$ bis $3,4 \mathrm{~cm} 1 / 10 \mathrm{HCl})$. Die höchsten Werte finden sich im Bereich des Angespüls. Im freien Wasser steigen die Werte von 2,0 auf $2,8 \mathrm{~cm} \mathrm{n/10} \mathrm{HCl}$, wobei die Zunahme wiederum auf abfließendes Porenwasser zurückzuführen ist. Im Bereich des Angespüls wird sogar ein Wert von 3,4 ccm erreicht.

Der Kalkgehalt des freien Wassers ist im allgemeinen etwas höher als der des Porenwassers, beispielsweise am 24. Mai 1965: $222 \mathrm{mg} \mathrm{CaO} / 1$ gegenüber 159 bis 
$177 \mathrm{mg} / \mathrm{lim}$ Porenwasser, oder am 1. Juni 1965: $236 \mathrm{mg} / 1$ gegenüber 181 bis $224 \mathrm{mg} / 1$ $\mathrm{CaO}$. Möglicherweise spielen bei den Schwankungen des Kalkgehaltes meteorologische Verhältnisse eine Rolle. Die Unterschiede im Gehalt von Magnesium im freien Wasser und Porenwasser sind viel ausgeprägter. Sie sind im freien Wasser um 150 bis $200 \mathrm{mg} / 1$ höher als im Interstitialwasser. Ob hierbei Ionen im Sand zurückgehalten werden, muß noch untersucht werden.

\section{BESPRECHUNG DER ERGEBNISSE}

Um einen Überblick über die chemischen Verhältnisse im Mesopsammal bei Cuxhaven und auch über die Wasser- und Bodenverhälnisse bei Helgoland zu erhalten, sind diese noch einmal in der Abbildung 8 zusammengestellt; sie zeigt den Strandabschnitt zwischen Tidenniedrig- und Tidenhochwasser. Die Zonen A bis $\mathrm{C}$ sollen den unterschiedlichen Wassergehalt im Lückensystem kennzeichnen.

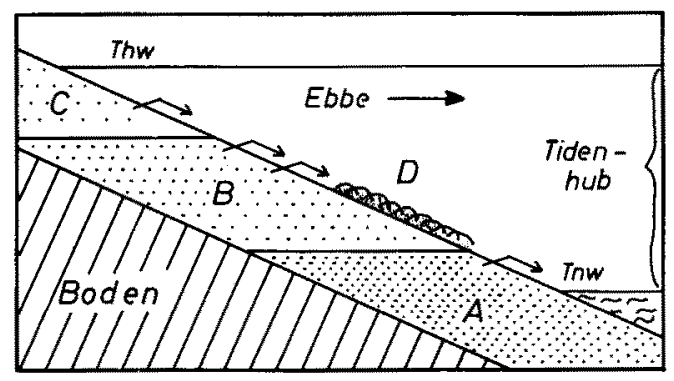

Abb. 8: Schematische Darstellung eines Strandes zwischen Tidenhochwasser (Thw) und Tidenniedrigwasser (Tnw). A, B, C: Schichten mit unterschiedlichem Gehalt an Porenwasser. D: Anwurf. Die Pfeile deuten das austretende Porenwasser bei Ebbe an. Nach Callame (1963) verändert

Bei Ebbe tritt Interstitialwasser aus den verschiedenen Horizonten aus und läuft den Hang hinunter und beeinflußt damit die darunterliegenden Wasserabschnitte. Besonders stark wird das Interstitialwasser in der Anwurfzone (D) beeinflußt, da hier der Kontakt $\mathrm{zwischen} \mathrm{Luft} \mathrm{und} \mathrm{Lückensystem} \mathrm{mehr} \mathrm{oder} \mathrm{weniger} \mathrm{stark} \mathrm{unterbunden} \mathrm{ist.} \mathrm{In}$ Richtung von $C$ nach A nimmt der Sauerstoffgehalt sehr stark $a b$, während die Zehrung zunimmt.

Ahnliches gilt auch für die anderen untersuchten chemischen Faktoren, wie zum Beispiel Nitrat und Phosphat. In allen Fällen ergaben sich beträchtliche Unterschiede in Interstitialwasser gegenüber dem darüber befindlichen freien Wasser. Bemerkenswert war auch, daß die chemischen Faktoren sich an einem Punkt der Bereiche C, B oder A im Verlauf einer Tide veränderten. Aus den gesamten Ergebnissen unserer Untersuchungen im Küstenbereich geht hervor, daß sich durch den ständig wechselnden Wasserstand im Verlauf der Tidenbewegung die chemischen Daten dauernd verändern. Hierin unterscheidet sich das Mesopsammal der Küstengebiete wesentlich von dem der bisher untersuchten Binnengewässer. WISZNIEwski (1934) und STANGENBERG (1934) 
haben durch ihre Untersuchungen gezeigt, daß das Mesopsammal ein hypereutrophes Milieu darstellt, das sich immer stärker mit Nährstoffen anreichert. Im Küstengebiet tritt durch die Tidenbewegung aber eine, wenn auch begrenzte, Auswaschung ein.

\section{ZUSAMMENFASSUNG}

1. An der "Muschelecke" vor dem Laboratorium in Cuxhaven wurden chemische Untersuchungen des Interstitialwassers (Mesopsammal) und des darüber befindlichen freien Wassers im Verlauf einer Tide durchgeführt.

2. Der Salzgehalt des Porenwassers war stets niedriger als der des freien Wassers. Im Verlauf einer Tide konnten an der gleichen Probenentnahmestelle geringe, aber deutliche Veränderungen festgestellt werden.

3. Der Sauerstoffgehalt des Interstitialwassers war stets niedriger als der des freien Wassers, die Sauerstoffzehrung erheblich größer. Der Sauerstoffgehalt nahm von den höheren Horizonten zu den tiefer gelegenen im Verlauf einet Tide deutlich $a b$, die Sauerstoffzehrung dagegen stieg an. Auch an jeder Probeentnahmestelle ging der Sauerstoffgehalt mit fallendem Wasser zurück.

4. Die Nitrat- und Phosphatgehalte des Porenwassers waren höher als die des freien Wassers. Das Gleiche galt für das Säurebindungsvermögen.

5. Im Porenwasser war der Gehalt an Kalk und Magnesium geringer als im freien Wasser.

\section{ZI'TIER'TE LIT'ERATUR}

Callame, B., 1963. Le milieu interstitiel dans les sediments sableux intercotidaux. Bull. Inst. ocúanogr. Monaco 60 (1271), 1-32.

Grknch, S., 1953. Die biocönotische Gliederung der Nematodenfauna an den Deutschen Küsten. Z. Morph. Ölol. Tiere 41, 411-512.

Kürn, H., 1952. Über die Hydrographie von Wattenpfützen. Helgoländer wiss. Meeresunters. $4,101-106$.

- 1962. Über die Schwankungen der abiotischen Faktoren in der Elbmündung bei Cuxhaven. Helgoländer wiss. Meeresunters. 10, 202-216.

- \& Mann, H., 1953. Beiträge zur Hydrochemic der Unterelbe. Veröff. Inst. Meeresforsch. Bremerh. 2, 236-268.

- 1956. Unperiodische Veränderungen im Stofthaushalt von Seewasseraquarien. Hydrobiologia 8, 66-78.

- 1966. Chemische Untersuchungen am Sandstrand von Cuxhaven und Helgoland. Veröf. Inst. Meeresforsch. Bremerh. (9) Sonderbd 2, 67-76.

RiemanN, F., 1966. Die Verbreitung der interstitiellen Fauna im Elbe-Aestuar. Veröf. Inst. Meeresforsch. Bremerh. (9) Sonderbd 2, 117-123.

Stangenberg, M., 1934. Psammolitoral, ein extrem eutrophes Wassermilicu. Arch. Hydrobiol. Ryb. 8, 274-284.

STowkl, F. P., 1927. The adsorption of ions from seawater by sand. J. mar. biol. Ass. U. K. $14,955-966$

WiszNiEwski, J, 1934. Recherches écologiques sur le psammon. J. mar. biol. Ass. U. K, 8, 149-271.

Deutsche Einheitsverfairren zur Wasseruntersuchung, 1960. 3. Aufl. Verl. Chemic Weinheim/Bergstr. 